\title{
Effect of Family Planning Counseling on Post Abortion Women Awareness and Practice of Using Contraceptive Methods
}

\author{
Walaa H. Ibrahim, Ihab M.H. El-Nashar, Entisar M. Youness \& Amal F. Arief. \\ Assistant lecturer of Obstetrics \& Gynecological Nursing, Faculty of Nursing, Assuit University, Egypt. \\ Professor of Obstetrics \& Gynecology Medicine, Faculty of Medicine, Assuit University, Egypt. \\ Assistant professor of Obstetrics \& Gynecological Nursing, Faculty of Nursing, Assuit University, Egypt. \\ lecturer of Obstetrics \& Gynecological Nursing, Faculty of Nursing, Assuit University, Egypt.
}

\begin{abstract}
Background: Unsafe abortion imposes heavy burdens on both individuals and society, particularly in low-income countries, many of which have restrictive abortion laws. Providing family planning counseling and services to women following an abortion has emerged as a key strategy to address this issue. The study aimed to assess the effect of family planning counseling on using contraceptive methods after abortion for women attending Woman's Health Hospital, Assiut University, Egypt. Methods: Quasi experimental design. Sample: convenient sample of 300 post aborted women divided into two groups A and B. Tools: A structured interviewing questionnaire used as a tool which included Sociodemographic, family planning history and follow up data Results: About $31.2 \%$ in group B versus $16.3 \%$ in group A used family planning method after abortion. Pills were the most method used between others methods in both groups $84.1 \%$ in group A and $68.2 \%$ in group B. Conclusion: women who had the family planning counseling about using contraceptive methods after abortion used family planning significantly higher than those in the non counseling group. Recommendations: Post abortion family planning counseling programs as an essential part of post abortion care is recommended \& must be generalized.
\end{abstract}

\section{Key Words : Post Abortive Counseling \& Contraceptive Methods.}

\section{Introduction}

This study was performed to identify the effect of family planning counseling on using of contraceptive methods after abortion. Abortion is the removal or expulsion of an embryo or fetus from the uterus, resulting in, or caused by its death (Ibisomi, 2008). Abortion is classified as either spontaneous or induced. Induced abortions are further subcategorized into two categories of therapeutic and elective abortions (Trupin, 2010).The number of abortions performed worldwide has remained stable in recent years 43.8 million having been performed in 2008 . The abortion ratio worldwide was 24 per 1000 women for developed countries and 29 per 1000 women for developing countries (Sedgh et al., 2012). In Assiut 2008 a study was utilized to determine the prevalence of abortion was found that $40.6 \%$ had aborted at least once; of them $24.6 \%$ had aborted more than once and were designated as recurrent abortion (Yassin, 2008).

There are two main methods of abortion surgical and medical. Surgical means the end of a pregnancy by surgically removing of embryo or fetus from a women's uterus. And this usually requires anesthesia to perform it (Kelli, 2013), But Medical abortion is a procedure that uses various medications to end the pregnancy (Mayo, 2013). For surgical abortion, women can leave the health-care facility as soon as they feel able and their vital signs are normal. Before leaving the health-care facility following the surgical abortion procedure, all women should receive contraceptive information and, if desired, the contraceptive method of their choice or referral for such services (Heidi, 2008).

Post-abortion family planning is the initiation and use of family planning methods immediately after, and within 48 hours of an abortion, before fertility returns. The provision of family planning is important for women in post-abortion periods because fertility can return surprisingly quickly after having an abortion. In some cases, women have become pregnant before having their first menstruation following abortion, and often the pregnancy is unwanted and may end up with a further abortion. Unfortunately, a large number of women who wish to delay or prevent future pregnancies receive little or no information on effective family planning methods post-abortion period, including how or where to obtain family planning methods, and how soon they should be started (Janice et al., 2011).

Family planning methods can be used immediately after post-abortion care, provided that there are no severe complications requiring further treatment, the provider screens for any precautions before using a particular contraceptive method, and the woman receives adequate counseling (Dougall, 2009). The nurse should advise women not to have sexual 
intercourse until their bleeding stops (usually five to seven days after abortion) and any complications have been resolved. Also advise women not to use natural family planning methods until their regular menstrual pattern returns (Janice et al., 2011).

All methods of contraception, including IUDs and hormonal contraceptives can be initiated immediately following surgical or medical abortion, as long as attention is paid to each woman's health profile and the limitations associated with certain methods. There are few methods that should not be started immediately following an abortion: the contraceptive diaphragm and cervical cap should not be used until 6 weeks after a second-trimester abortion, and fertilityawareness-based methods should only be started after the resumption of regular menses (WHO, 2008). All women should be informed about emergency contraception, and consideration should be given to providing it to them to be kept at home for future use, particularly for women who choose condoms as their primary method of contraception (WHO, 2012).

\section{Significant of the study}

A study was utilized in Assuit to determine the prevalence of abortion was found that $40.6 \%$ had aborted at least once; of them $24.6 \%$ had aborted more than once and were designated as recurrent abortion (Yassin, 2008). The provision of family planning is important for women in post-abortion periods because fertility can return surprisingly quickly after having an abortion. Immediate pregnancy may be contraindicated on medical grounds. Post abortion family planning is therefore an integral part of comprehensive abortion care to avoid an overreliance on abortion for preventing unwanted pregnancies. Offering contraceptive information, services and referrals with abortion services for unwanted pregnancies can reduce the consequent need for abortion (Mittal, 2006). So the investigator interested to identify the effect of post abortive family planning counseling on using contraceptive methods.

\section{Aim of the study}

This study aimed to assess the effect of family planning counseling on using contraceptive methods after abortion for women attending Woman's Health Hospital at Assiut University.

Research question

Did family planning counseling affect the use of contraceptive methods after abortion?

Subjects and methods

Research design: Quasi experimental design was utilized in this study.

\section{Setting}

The study was conducted at the post abortion ward, Woman's Health Hospital, Assiut University, Egypt. This ward received post abortion women who are seeking the care after abortion.

\section{Sample}

According to sample size equation the sample included 284 women and increased to 300 women (convenient sample) that admitted to the Woman's Health Hospital at Assiut University for abortion. These women were divided into two groups. Group A, which received routine hospital care, (150 women) and groups group $\mathrm{B}$, which received family planning counseling, (150 women). There was dropped out of 15 women in group A and 9 in group B during follow up phase.

\section{The routine hospital post abortive care}

It started when the woman discharged from the operating room, the anesthetist ensured that her state is being stable, she is oriented and her blood pressure within normal, she transferred to the post abortive ward for treatment.

The woman receives intravenous fluids, oxytocin (30 units) and the prescribed oral antibiotics after arriving to the recovery room. The nurse gives her clean pad and checking the amount of blood loss. If there were any abnormalities like postpartum hemorrhage or fever the nurse informs the Obstetrician and the case receives treatment as prescribed.

In the second day abortion the woman discharges and receives a discharge card that contained the treatment. Discharge occurs after examination of the woman by the Obstetrician and be ensured that the case is good, no vaginal bleeding, no fever, no investigation need, and no complain. After that the woman would be asked to come to post abortive outpatients clinics after two weeks for follow up to check if there were any complications occurred to the woman during this period.

Tools

A Structured interviewing questionnaire used in the current study which included the following data:

Sociodemographic data: (Name, age, address, educational level, residence and occupation)

Obstetric history: (Gravidity, parity, abortions, stillbirths, neonatal deaths and number of living children.

Data related to family planning history: (Previous use of family planning methods, duration of use, cause of discontinuation)

\section{Data related to the current abortion}

(Duration of pregnancy, type of abortion and the intention of these women for the use of family planning methods) 
Follow up data: Data related to using family planning methods during follow up period, types of family planning methods used and the cause of not use.

\section{The Procedure}

\section{Administrative phase}

Before implementation of the study, an official permission obtained from the Dean of the Faculty of Nursing then directed to the director of Woman's Health Hospital, Assiut University, Egypt after full explanation of the aim of the study.

\section{Pilot study}

The pilot study was carried out on 10\% (30 women) before implementation of the study to test the validity and reliability of the tools. The necessary modifications were done based on the results of the pilot study. Women who participated in the pilot study were not included in the main study.

The investigator interviewed the post abortion women at the post abortion ward, Woman's Health Hospital, Assiut University, Egypt. Random assignment was done by computer generated tables. Concealed envelopes containing the type of care received to the women that opened before deciding to include the case in the study. The investigator chose one envelop randomly to determine the woman included in group A or B.

\section{Field work}

The data were collected in 14 months from 20/1/2013 to $10 / 3 / 2014$.

Intervention phase

The investigator introduced her to the women and explained the nature of the study to obtain an oral consent from them to participate in the study. The investigator met the women in post abortion ward. Then collected the questionnaire which included the sociodemographic characteristics such as name, age, education and occupation. The obstetric history such as gravidity, parity, abortion, and other issues related to obstetric history. Then the investigator collected data related to family planning history such as previous use of family planning methods, duration of use, cause of discontinuation. The data related to the current abortion such as duration of pregnancy, type of abortion and the intention of these women for the future use of family planning methods. The last part of the questionnaire was focused on group B which received counseling about family planning methods that included time of fertility return, time of using methods, and explanation of various methods used, its advantages and disadvantages. The average time taken for filling each tool ranged from 15-20 minutes depending on the response of the woman. In addition to 15 minute to give family planning counseling for group B only. Then the investigator followed the women after two weeks to saw if they used one of the contraceptive methods or not and cause of not using.

\section{Ethical consideration}

The study protocol was approved by pertinent research and ethics committees. No harmful procedure was affected on the condition of the mother. Oral informed consent was taken from every woman before inclusion in the study. Each woman was allocated to the proper group according to the study profile. A code number was used for every woman to maintain confidentiality.

\section{Statistical Analysis}

Data entry and statistical analysis were done using Statistical Package for the Social Sciences (SPSS, version 16). Comparison between the groups was done using Student's t-test to compare the mean values between groups in scale variables. For analysis $\mathrm{P}<0.05$ was considered significant. 


\section{Results}

Table (1) : Distribution of the studied women according to their sociodemographic characteristics.

\begin{tabular}{|l|c|c|c|c|c|}
\hline \multirow{2}{*}{ Sociodemographic characteristics } & \multicolumn{2}{|c|}{ Group A N=(150) } & \multicolumn{2}{|c|}{ Group B N=(150) } & \multirow{2}{*}{ P-value } \\
\cline { 2 - 5 } & $\mathbf{N o}$ & $\mathbf{( \% )}$ & $\mathbf{N o}$ & $\mathbf{( \% )}$ & \\
\hline 1) Age/years & & & & & \\
15-less than 20 years & 19 & 12.7 & 18 & 72.7 & 0.971 \\
20-35 years & 111 & 74 & 109 & 15.3 & \\
More than 35 years & 20 & 13.3 & 23 & & \\
\hline 2) Occupation & & & & & \\
Housewife & 140 & 93.3 & 139 & 92.7 & \\
Employed & 10 & 6.7 & 11 & & \\
\hline 3) Residence & & & & & \\
Urban areas & 44 & 29.3 & 50 & 33.3 & 0.532 \\
Rural areas & 106 & 70.7 & 100 & 66.7 & \\
\hline 4) Educational level & & & & & \\
Illiterate\& read and write & 48 & 32 & 43 & 28.7 & \\
Primary and elementary & 29 & 19.3 & 31 & 20.7 & 0.265 \\
Secondary & 57 & 38 & 47 & 31.3 & \\
University & 16 & 10.7 & 29 & 19.3 & \\
\hline
\end{tabular}

Table (2): Distribution of the studied women according to family planning history.

\begin{tabular}{|c|c|c|c|c|c|}
\hline \multirow[b]{2}{*}{ Variables } & \multicolumn{2}{|c|}{ Group A } & \multicolumn{2}{|c|}{ Group B } & \multirow[b]{2}{*}{ P-value } \\
\hline & No & $(\%)$ & No & $(\%)$ & \\
\hline $\begin{array}{l}\text { 1) Previous using of contraceptive method } \\
\text { Used }\end{array}$ & 68 & 45.3 & 66 & 44 & \multirow{3}{*}{0.742} \\
\hline Not used & 82 & 54.7 & 84 & 56 & \\
\hline Total & 150 & 100 & 150 & 100 & \\
\hline 2) Last method used & & & & & \multirow{7}{*}{0.230} \\
\hline Pills & 33 & 48.5 & 32 & 48.5 & \\
\hline IUD & 15 & 22.1 & 21 & 31.8 & \\
\hline Injectables & 19 & 27.9 & 9 & 13.6 & \\
\hline Implanon & 1 & 1.5 & 3 & 4.6 & \\
\hline Barrier method & --- & ----- & 1 & 1.5 & \\
\hline Total & 68 & 100 & 66 & 100 & \\
\hline 3) Cause of stopping use & & & & & \multirow{6}{*}{0.151} \\
\hline Pregnant while using & 12 & 17.7 & 5 & 7.6 & \\
\hline Wanted to get pregnant & 30 & 44.1 & 24 & 36.4 & \\
\hline Side effect & 24 & 35.3 & 31 & 46.9 & \\
\hline Others \# & 2 & 2.9 & 6 & 9.1 & \\
\hline Total & 68 & 100 & 66 & 100 & \\
\hline
\end{tabular}

\# (expired date, traveling of husband) 
Table (3): Distribution of the studied women according current pregnancy data.

\begin{tabular}{|l|c|c|c|c|c|}
\hline \multirow{2}{*}{ Variables } & \multicolumn{2}{|c|}{ Group A N= (150) } & \multicolumn{2}{c|}{ Group B N= (150) } & \multirow{2}{*}{ P-value } \\
\cline { 2 - 5 } & No & $\mathbf{( \% )}$ & No & $\mathbf{( \% )}$ & \\
\hline 1) Desire toward this pregnancy & & & & & \multirow{2}{*}{0.242} \\
Wanted pregnancy to continue & 116 & 77.3 & 108 & 72 & \\
Unwanted pregnancy & 34 & 22.7 & 42 & 28 & \\
\hline 2) Desire to get pregnant again & & & & & \multirow{2}{*}{0.378} \\
Yes & 121 & 80.7 & 115 & 76.7 & \\
No & 29 & 19.3 & 35 & 23.3 & \\
\hline 3) Duration of pregnancy & & & & & \\
First trimester abortion & 98 & 65.3 & 110 & 73.3 & 0.249 \\
Second trimester abortion & 52 & 34.7 & 40 & 26.7 & \\
\hline 4) Type of abortion & & & & & \\
Missed & 50 & 33.3 & 53 & 35.3 & \\
Inevitable & 76 & 50.7 & 75 & 50 & 0.685 \\
Incomplete & 24 & 16 & 21 & 14 & \\
Septic & 0 & ---- & 1 & 0.7 & \\
\hline
\end{tabular}

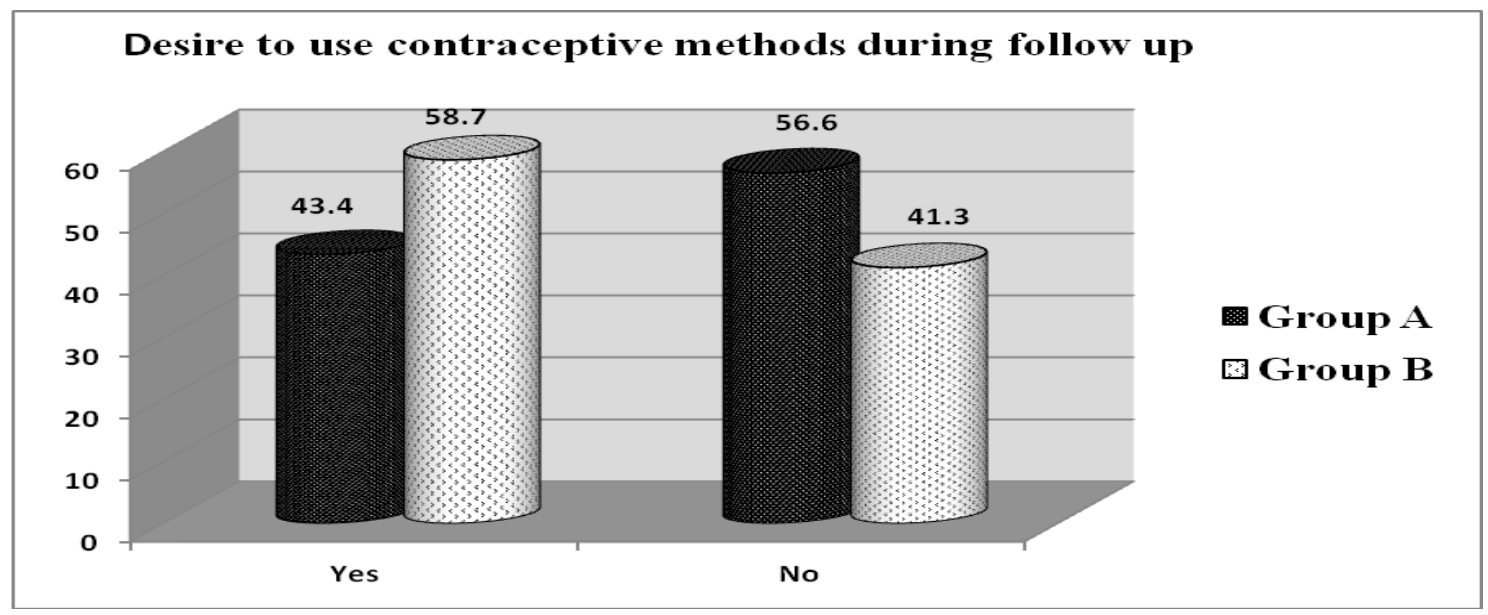

Figure (1): Desires to use contraceptive method during follow up.

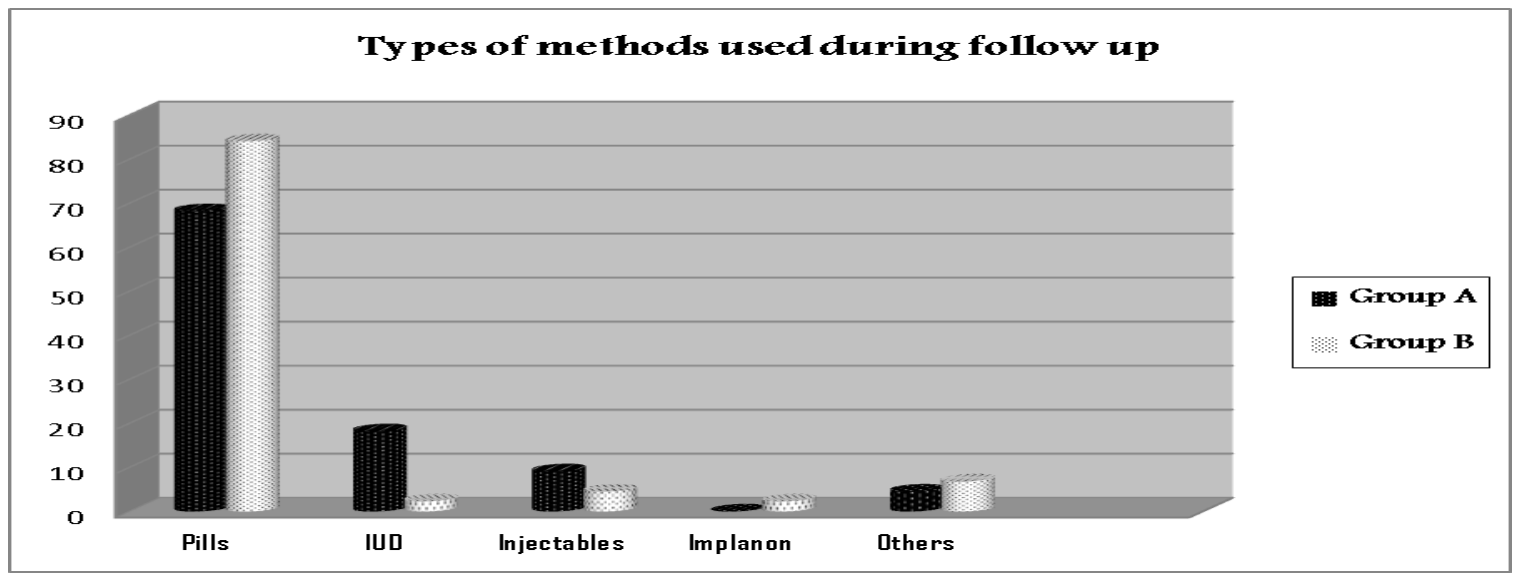

Figure (2): Type of contraceptive method used during follow up. 
Table (4): Desire to get pregnant in relation to using family planning methods within two weeks after discharge.

\begin{tabular}{|c|c|c|c|c|c|}
\hline \multirow{3}{*}{ Using family planning } & \multicolumn{4}{|c|}{ Desire to get pregnant } & \multirow{3}{*}{ P-value } \\
\hline & \multicolumn{2}{|c|}{ Yes } & \multicolumn{2}{|c|}{ No } & \\
\hline & Group A(135) & Group B(141) & Group A(135) & Group B(141) & \\
\hline $\begin{array}{l}\text { Yes } \\
\text { No }\end{array}$ & $\begin{array}{c}12(8.9 \%) \\
96(71.1 \%)\end{array}$ & $\begin{array}{c}30(21.3 \%) \\
79(56 \%)\end{array}$ & $\begin{array}{c}11(8.1 \%) \\
16(11.9 \%)\end{array}$ & $\begin{array}{c}14(9.9 \%) \\
18(12.8 \%)\end{array}$ & \multirow[t]{2}{*}{0.000} \\
\hline Total & $108(80 \%)$ & $109(57.3 \%)$ & $27(20 \%)$ & $32(22.7 \%)$ & \\
\hline
\end{tabular}

Table (5): Desire to use family planning methods after abortion in relation to using family planning methods within two weeks after discharge.

\begin{tabular}{|c|c|c|c|c|c|}
\hline \multirow{3}{*}{ Using family planning } & \multicolumn{4}{|c|}{ Desire to use family planning methods } & \multirow{3}{*}{ p-value } \\
\hline & \multicolumn{2}{|c|}{ Yes } & \multicolumn{2}{|c|}{ No } & \\
\hline & Group A(135) & Group B(141) & Group A(135) & Group B(141) & \\
\hline $\begin{array}{l}\text { Yes } \\
\text { No }\end{array}$ & $\begin{array}{l}18(13.3 \%) \\
39(28.9 \%)\end{array}$ & $\begin{array}{l}39(27.7 \%) \\
43(30.5 \%)\end{array}$ & $\begin{array}{c}4(2.96 \%) \\
74(54.8 \%)\end{array}$ & $\begin{array}{c}5(3.5 \%) \\
54(38.3 \%)\end{array}$ & \multirow{2}{*}{0.000} \\
\hline Total & $57(42.2 \%)$ & $82(58.2 \%)$ & $78(57.8 \%)$ & $59(41.8 \%)$ & \\
\hline
\end{tabular}

Table (1): shows that there is no significant differences among women of both groups regarding age groups. Almost all of them in both groups are housewives. As regards residence, $(70.7 \%$ and $(66.7 \%)$ in group A and B respectively are from rural areas with no significant statistical differences. According to education 38\% in group A and $31.3 \%$ in group B are had secondary level of education.

Concerning contraceptive profile among both groups

Table (2) : shows that more than one half of women in both groups have not use any contraceptive method before From the last method used, pills take the more percent $48.5 \%$ in both group, but $1-3$ years of using last method take more than one third of women. As regard stopping use of the contraceptive methods more than one third of women in both groups leave the contraceptive method because they want to get pregnant.

Regarding desire of women to use contraceptive method after abortion

Figure (1): reports that $(43.4 \%)$ in group A and $(58.7 \%)$ in group B want to use contraceptive method after abortion with statistical significant differences between both groups p-value (0.007).

Table (3): shows that around three quarters of women in both groups are wanted pregnancy to continue. More than three quarters of women want to get pregnancy again in both groups. About $(65.3 \%)$ of women in group A and (73.3\%) in group B had their abortion in first trimester.

Figure (2): shows that pills are the most method used between others methods in both groups.

Table (4): identifies that there is significant difference between both groups in women desired to get pregnant regarding using family planning methods after abortion p-value (0.000).

Table (5): illustrates that there is significant difference between both groups relation between desire to use family planning methods after abortion and using family planning methods after abortion $\mathrm{p}$ value (0.000).

\section{Discussion}

Regarding maternal age, the present study demonstrated that the majority of women in the age group 20-35 years. This was disagreed with Ali et al., (2009), who performed his study in DiyarbakirTurkey to assess Post abortion family planning counseling as a tool to increase contraception use who found that more than half $(56.9 \%)$ and Ana et al., (2010). who performed their study in the northeast Brazil about choices on contraceptive methods in post-abortion family planning clinic found that as nearly half (46.7\%) of aborted women were employed as different in culture of the country. The present study revealed that the great majority of women were housewives and more than two thirds of them were lived in rural areas. In the same line, these findings were consistent with Ali (2007). who showed that the great majority of women $(93.8 \%)$ were housewives and more than two thirds of them were lived in rural areas (74.8\%). These findings were nearly agreed with Mahmoud \& Byomy (2013). who reported that the great majority of women $(96.2 \%)$ were housewives and the majority of them lived in rural areas (90\%). This consistency was related to similarity between these in the place of performing the study as all of them performed in Upper Egypt. 
While Ana et al., (2010). who performed their study in the northeast Brazil about choices on contraceptive methods in post-abortion family planning clinic showed that $(46.7 \%)$ of aborted women were employed and Rodrigo et al., (2011). who mention in their study factors that associated with the occurrence of abortion in Brazil they found that, the great majority of aborted women (90.9\%) lived in urban areas. This disagreement may be due to difference in culture between Brazil and Egypt.

Regarding educational level the present study reported that (38\% in group A and $31.3 \%$ in group B) had secondary education. This this is nearly agreed with Shyam \& Shailes (2013). who performed their study in Nepal to show risk factors for repeated abortion, they found that $(38.5 \%)$ of aborted women had secondary education. This finding was disagreed with Ali et al., (2009). who showed that (12.2\%) of aborted women had secondary education. Also Rodrigo et al., (2011). study reported that (21.6\%) of aborted women had high secondary school. This percent of secondary educational level (38\% in group $\mathrm{A}$ and $31.3 \%$ in group B) explain increased percent of marriage in the age group (15-20) years $(56 \%$ in group A versus $46.7 \%$ in group B) and this shows that high percent of females married after finishing secondary education.

Post abortion family planning is therefore an integral part of comprehensive abortion care to avoid an overreliance on abortion for preventing unwanted pregnancies. Offering contraceptive information, services and referral with abortion services for unwanted pregnancies can reduce the consequent need for abortion (Mittal, 2006).

Regarding previous uses of contraceptive methods, the present study revealed that more than half in both group of women were not used any type of contraceptive methods. From the last method used, pills took the highest percent $48.5 \%$ in both groups. It is also showed that $36.7 \%$ in group A versus $42.4 \%$ in group B were used contraceptive methods for a duration from (1-3) years and $44.1 \%$ in group $\mathrm{A}$ and more than one third of women $(36.4 \%)$ in group B terminated the contraceptive method because they wanted to get pregnant. These findings were nearly agreed with Mahmoud \& Byomy (2013). who found that more than half $(68.6 \%)$ of aborted women were non users. The majority $(81.8 \%)$ of women used contraceptive methods for a duration from (1-3) years, and $(43.6 \%)$ terminated the use of methods to get pregnant. Incongruent with these findings corinne et al., (2014). who study the use of post abortive contraceptives in Nepal, they found that $39.9 \%$ of aborted women were not used any type of contraceptive methods. Also Michele et al., (2013). in their study about Induced abortion, contraceptive use, and dual protection among female sex worker in Moscow, Russia reported that $2.1 \%$ only were not used any type of contraceptive methods. Moreover, Mahesh et al., (2011). who applied their study in Nepal to identify the post abortive contraceptive use, illustrated that over half $(57 \%)$ of aborted women had previously used pills or injectables and 6\% used IUD or implanon. It was also found that the most frequently mentioned reasons for discontinuations of previously used contraceptives were fear of side effects or health concerns $(33 \%)$ followed by inconvenient to use (24\%) and then husband was away (17\%).Increased percent of women who are non users for contraceptive methods may be related to lack of interest in counseling about contraceptive methods.

Most women who experience abortion, especially induced abortion of an unintended pregnancy, do not desire to become pregnant immediately. In a few circumstances، immediate pregnancy may be contraindicated on medical grounds. Post abortion family planning is therefore an integral part of comprehensive abortion care to avoid an overreliance on abortion for preventing unwanted pregnancies. Offering contraceptive information, services and referrals with abortion services for unwanted pregnancies can reduce the consequent need for abortion (Mittal, 2006).

According to desires to use contraceptive methods after abortion the present study reported that $43.4 \%$ in group A versus $58.7 \%$ in group B were intended to use contraceptive method after abortion. This explains the importance of post abortive counseling about contraceptive methods as increased percent of group B than A. These findings were nearly agreed with Mahmoud \& Byomy (2013). who found that $37.1 \%$ were intended to use contraceptive method after abortion.

Concerning current pregnancy data, the present study showed that $(77.3 \%$ in group A and $72 \%$ in group B) of women were wanted pregnancy to continue. More than three quarters of women $(80.7 \%$ in group A and $76.7 \%$ in group B) were desired to get pregnancy again. In the same line Mahmoud \& Byomy (2013). reported that more than three quarters of aborted women $(76.2 \%)$ wanted pregnancy to continue. Also Ali (2007) found that the majority of aborted women $(87.6 \%)$ wanted pregnancy to continue. This disagreement may be related to the difference in culture between Egypt, Nepal and Brazil. On the other hand, Corinne et al., (2014) and Ana et al., (2010) illustrated that (40.9\%) and (48\%) of aborted women desired to get pregnancy again. And a small percent $(16.5 \%)$ intended to get pregnant in the future found in the study of Shyam \& shailes (2013). on post aborted women. Also Mahesh et al., (2011). 
showed that more than half $(59 \%)$ of aborted women didn't want another child. This disagreement may be related to differences in culture between countries. In dealing with follow up data, it was a dropped out of 15 cases in control group versus 9 cases in cases group, so the women in control group enumerated 135 women and 141 women in case group.

The majority of women $(82.8 \%)$ had spontaneous abortion while $17.2 \%$ had induced abortion. For women who wish to postpone or prevent future pregnancies, it is important to begin using a contraceptive method immediately after abortion (Mittal, 2006).

As regard using contraceptive methods during follow up phase, the present study illustrated that $(16.3 \%)$ of women in group A versus (31.2\%) in group B used contraceptive methods during follow up period, $28.3 \%$ in group A versus $23.3 \%$ in group B explained not using contraceptive methods by waiting for the next period to use contraceptive method. This explains the role of family planning counseling that given to post aborted women through the investigator. These findings were congruent with Mahmoud \& Byomy (2013), who showed that $62.9 \%$ of aborted women didn't intend to use contraceptive methods after abortion. But, the vast majority of them $(90.1 \%)$ refused to use contraceptive methods because they want to get pregnant again. On the other hand, Thoai et al., (2014). in their study about risk factors for repeat abortion and implications for addressing unintended pregnancy in Vietnam, reported that the majority of aborted women $(85.4 \%)$ wanted to use contraceptive methods after abortion. Also Shyam \& shailes (2013). showed that the majority $(77.5 \%)$ of aborted women were intended to use contraceptive methods after abortion. This may be related to the importance of these countries in giving family planning counseling as a routine care after abortion.

Regarding type of contraceptive method used after abortion, the present study revealed that oral contraceptive pills were the most method used after abortion (68.2\% in group A versus $84.1 \%$ in group B) followed by IUD (18.2\% in group A versus $2.3 \%$ in group B).This was incongruent with Ana et al., (2010). who found that the most common contraceptive method used after abortion was injectable $(50 \%)$ followed by oral contraceptive pills (2.3\%). Another study by Shrestha \& Sharma, (2013) reported that among post aborted women who accepted contraceptive methods, Dept provera was the highest $(22.8 \%)$ followed by oral contraceptive pills $(19.6 \%)$

The present study demonstrated that there was relation between using post abortive contraceptive methods and desired (toward this pregnancy, to get pregnant, and to use contraceptive methods) with $\mathrm{p}$ value (0.000).

\section{Conclusion}

Family planning counseling after abortion had significant effect on increasing the percent of using contraceptive methods during follow up period.

\section{Recommendations}

Post abortion family planning counseling programs as an essential part of post abortion care is recommended \& must be generalized. Health care providers should increase the awareness of post abortion women about the time of fertility return. More researches should be done to assess the impact of post abortion family planning counseling on reducing the rate of unwanted pregnancy.

Acknowledgements

We would like to express our deep appreciation to all patients who participate in succession my research. We would also like to thank the medical and nursing staffs who participate in highlighting the aims of my research.

\section{References}

1. Ali S., Ahmed (2007) : the incidence of post abortive danger signals that denote physical and psychological complications, p 55-87

2. Ana Laura C., Ferreira, Ariani I., Souza, Raitza A., Lima \& Cynthia Braga (2010): choices on contraceptive methods in postabortion family planning clinic in the northeast Brazil, reproductive-health-journal 112(2): 1-5.

3. Corinne H., Rocca, Mahesh Puri, Cynthia C., Harper, Maya Blum, Bishnu Dulal, Jillian T., Henderson (2014): Post abortion contraception a decade after legalization of abortion in Nepal, International Journal of Gynecology and Obstetrics xxx 118(7): 1-5.

4. Heidi Baker (2008): First trimester abortion Guidelines and protocols, Surgical and medical procedures, Published by the International Planned Parenthood Federation (IPPF), Accessed August 23.

5. Ibisomi L., (2008): Pregnancy termination in sub-Saharan Africa: the need for refined data. International Journal of Health Research; 110(23): 207-224.

6. Janice Tripney, Karen Schucan Bird, Irene Kwan \& Josephine Kavanagh (2011) : The impact of post-abortion care family planning counseling and services in low-income countries: a systematic review of the evidence, 
International Journal of Gynecology and Obstetrics 113(5): 212-214.

7. Kelli Bamforth, (2013): eHow, Family Health, Family Planning Information, Abortions, What Is a Surgical Abortion?, Harvard University Press. ISBN 978-0-674-27024-4. OCLC 36126503.

8. Mahesh Puri, Corinne Rocca, Maya Blum, Cynthia Harper, Deepak Joshi \& Jillian Henderson (2011): Post-abortion contraceptive use and continuation in Nepal, http://www.reproductive-healthjournal.com/content 113(9): 1-5.

9. Mahmoud Ghadah A., \& Byomy Soad S., (2013): Fertility awareness and family planning use among post abortion women in Egypt, Life Science Journal;10(1) :143-150 http://www.lifesciencesite.com.

10. Mayo Clinic staff (2013): Mayo Foundation for Medical Education and Research (MFMER) "MayoClinic.com," definition of medical abortion, available at MayoClinic.com on 15 September.

11. McDougall J., Fetters T., Clark K., Rathavy T., (2009): Determinants of contraceptive acceptance among Combodian abortion patient; 40(2): 123 .

12. Michele R., Decker, Eileen A., Yam, Andrea L., Wirtz, Stefan D., Baral, Alena Peryshkina, Vladmir Mogilnyi, Chris Beyrer (2013): Induced abortion, contraceptive use, and dual protection among female sex workers in Moscow, Russia, International Journal of Gynecology and Obstetrics 120 (4): 27-31.

13. Mittal, S., (2006): Contraception after medical abortion, Contraception 74p56-60.

14. Rodrigo S., Camargo, Danielly S.. Santana, José G., Cecatti, Rodolfo C., Pacagnella, Ricardo P., Tedesco, Elias F., Melo J., Maria H., Sousa (2011): Severe maternal morbidity and factors associated with the occurrence of abortion in Brazil, International Journal of Gynecology and Obstetrics 112(8): 88-92.

15. Sedgh, G., Singh, S., Shah, I., Åhman, E., Henshaw, S., Bankole, A., (2012) : "Induced abortion: Incidence and trends worldwide from 1995 to 2008". The Lancet 379 (9816):625632.Doi:10.1016/S0140-6736(11)61786

8. PMID 22264435.

16. Shrestha A., \& Sharma P., (2013): Post Abortion Choice and Acceptance of Contraception, original article, NJOG|8 (1)ISSUE 15 |Jan-Jun,.

17. Shyam Thapa, Shailes Neupane (2013): Risk factors for repeat abortion in Nepal, International Journal of Gynecology and Obstetrics 120(5): 32-36.
18. Thoai D., Ngo, Sarah Keogh, Thang H., Nguyen, Hoan T., Le, Kiet H., Pham, Yen B., Nguyen (2014): Risk factors for repeat abortion and implications for addressing unintended pregnancy in Vietnam, International Journal of Gynecology and Obstetrics. 125(24): 241-246.

19. Trupin S., (2010): Elective abortion, Classification of abortion. Retrieved from: http://emedicine.medscape.com/article/252560overview,_assessed on May 27.

20. World Health Organization (2008): post abortion care family planning counseling: A Guide for Midwives and Doctors: World Health Organization. ISBN 978-92-4-1545877. OCLC 181845530.

21. World Health Organization (2012): Safe Abortion, 2nd edition, Technical and Policy Guidance for Health Systems, Clinical care for women undergoing abortion, available from the origin, Accessed April 14.

22. K., Yassin (2008): Incidence and socioeconomic determinants of abortion in rural Upper Egypt, 114(4): 269-272.

23. Ali Ceylan, Meliksah Ertem, Gunay Saka \& Nurten Akdeniz (2009): Post abortion family planning counseling as a tool to increase contraception use, BMC Public Health, 10(118): 9-20. 